\title{
Transglutaminase 2 Accelerates Vascular Calcification in Chronic Kidney Disease
}

\author{
Neal X. Chen ${ }^{a}$ Kalisha O'Neilla Xianming Chen ${ }^{a}$ Kraiwiporn Kiattisunthorn ${ }^{c}$ \\ Vincent H. Gattone ${ }^{a}$ Sharon M. Moe ${ }^{a, b}$ \\ andiana University School of Medicine, and ${ }^{b}$ Roudebush VA Medical Center, Indianapolis, Ind., USA; \\ 'Faculty of Siriraj Medical School, Mahidol University, Bangkok, Thailand
}

\section{Key Words}

Vascular calcification - Extracellular matrix-cell interaction .

Chronic kidney disease

\begin{abstract}
Background: Transglutaminase 2 (TGM2) is a calcium-dependent enzyme that can cross-link nearly all extracellular matrix (ECM) proteins and can facilitate cell-ECM interaction through integrins. Given the importance of the ECM in vascular calcification we tested the hypothesis that increased TGM2 activity may accelerate vascular calcification in chronic kidney disease (CKD). Methods: We utilized thoracic aortas and vascular smooth muscle cells (VSMC) from the Cy/+ rat, a model of progressive CKD that develops arterial calcification on a normal phosphorus diet, compared to normal rats. Results: VSMC isolated from CKD rats had increased expression and activity of TGM2 compared to cells from normal rats. The increased calcification and expression of alkaline phosphatase activity observed in VSMC from CKD rats compared to normal was inhibited in a dose-dependent manner with the TGM inhibitors cystamine and Z006. Matrix vesicles (MV) from CKD rat VSMC also had increased TGM2 expression and the calcification of MV on type I collagen could be inhibited with cystamine and accelerated by exogenous cross-linking of fibronectin or type I collagen with TGM2. Finally, the calcification of aorta rings from CKD rats in ex vivo
\end{abstract}

cultures was inhibited with TGM2 inhibitor. Conclusion: These data demonstrate a role of TGM2 in the pathogenesis of vascular calcification in CKD through enhancement of MVECM calcification.

Copyright $\odot 2013$ S. Karger AG, Basel

\section{Introduction}

Patients with chronic kidney disease (CKD) are more likely to die of a cardiovascular event than reach the need for dialysis. A major cause of cardiovascular morbidity and mortality in CKD is increased coronary artery and peripheral calcification. The pathogenesis is multifactorial, but on a cellular level it resembles normal bone mineralization [1]. The pathophysiology of vascular calcification is complex but several lines of evidence support that (1) vascular smooth muscle cells (VSMC) trans-differentiate to an osteoblast-like phenotype through upregulation of 'bone'-specific transcription factors, (2) the transdifferentiation occurs in response to a combination of hyperphosphatemia and other uremic toxins, including hypercalcemia or increased calcium load, (3) these transformed VSMC then lay down an extracellular matrix (ECM) that is subsequently remodeled by matrix metalloproteinase, priming the ECM for subsequent calcification, and (4) the cells then mineralize the ECM by the 
formation of matrix vesicles (MVs), small (50-200 nm) calcium-phosphorus-rich exosomes. This initiation and propagation of calcification can occur even in the absence of additional cells $[1,2]$. We have characterized the content of the MV and demonstrated that increased annexinII and decreased fetuin-A content leads to increased ability to mineralize ECM proteins [3]. Furthermore, the formation of these MV requires an influx of calcium (extracellular to intracellular) and was dependent on signaling pathways involved in cytoskeleton structure and organization $[4,5]$.

Transglutaminase 2 (TGM2) is a calcium-dependent enzyme that is a co-receptor with $\beta 1$ integrin and fibronectin on the cell membrane, and therefore involved in the communication between the cytoskeleton and the ECM. TGM2 is also located intracellular, and when activated in response to increased intracellular calcium, can bind proteins thereby altering their subcellular localization. Finally, TGM2 can cross-link nearly all ECM proteins by posttranslational modification through transamidation or deamidation [6]. Such alterations in the ECM increase osteoblast differentiation [7] and alter arterial remodeling in cell culture and non-CKD animal models [6] suggesting a pathologic role in bone and cardiovascular disease. Furthermore, VSMC from TGM2-null mice cannot calcify in response to hyperphosphatemia [8]. The current study is to test our hypothesis that increased TGM2 in CKD accelerates vascular calcification.

\section{Materials and Methods}

\section{Cell Culture}

Primary rat VSMC (RVSMC) were isolated from the descending thoracic aorta in normal or Cy/+ (CKD) rats (between 25-30 weeks old) by the explant method as previously described [5] and grown in Dulbecco's Modified Eagles Medium (DMEM; Sigma, St. Louis, Mo., USA), with 10\% FBS. To induce calcification, RVSMC were treated with $5 \mathrm{mM} \beta$-glycerophosphate, $1 \mathrm{U} / \mathrm{ml}$ fetal alkaline phosphatase (ALP), $10^{-7} \mathrm{M}$ insulin and $50 \mu \mathrm{g} / \mathrm{ml}$ ascorbic acid in the presence of $15 \%$ serum [9]. In some experiments, RVSMC were also treated with the TGM2 inhibitor cystamine (Sigma, St. Louis, Mo., USA) or specific TG2 inhibitor Z006 (Zedira GmbH, Darmstadt, Germany) [10].

\section{Real-Time (Quantitative) RT-PCR Analysis}

Total RNA was isolated from RVSMC using Trizol Reagent (Invitrogen, Carlsbad, Calif., USA). The TGM2 and RUNX2 expression in RVSMC was determined by real-time PCR using $2 \mu \mathrm{g}$ of total RNA in TaqMan Reverse Transcription reagent (Applied Biosystems, Foster City, Calif., USA). Target-specific PCR primers for TGM2 and RUNX2 were obtained from Applied Biosystems. Real-time PCR amplification was performed using TaqMan Gene Expression Assays (TaqMan MGP probes, FAM dye-labeled) us- ing Applied Biosystems 7500 Real-Time PCR system (Applied Biosystems). The cycle number at which the amplification plot crosses the threshold was calculated $\left(\mathrm{C}_{\mathrm{T}}\right)$, and the $\Delta \Delta \mathrm{C}_{\mathrm{T}}$ method was used to analyze the relative changes in gene expression using $\beta$-actin as a housekeeping gene.

\section{Cellular TGM2 Activity}

For cellular TGM2 activity measurements, incorporation of 5-(biotinamido) pentylamine (BAP; Sigma, St. Louis, Mo., USA) into proteins was determined in normal or CKD RVSMC using published methods [11]. After cell culture, VSMC were washed with phosphate-buffered saline (PBS) and then incubated with culture media containing $1 \mathrm{mM}$ BAP for $4 \mathrm{~h}$. The cell lysate was isolated using 1\% NP-40 buffer (50 mM TrisHCl, pH 7.4; 5 mM DTT; $50 \mathrm{mM} \mathrm{KCL} ; 3 \mathrm{mM}$ EDTA; $5 \mathrm{mM} \mathrm{MgCl}_{2} ; 150 \mathrm{mM} \mathrm{NaCl}$ ) and the protein concentration of the lysates was determined with the BCA assay. For detection of TGM2 activity, 96-well Maxisorp plates were incubated with $50 \mu \mathrm{l}$ of coating buffer $(50 \mathrm{mM}$ Tris, $150 \mathrm{mM}$ $\mathrm{NaCl}, 5 \mathrm{~mm}$ EGTA, 5 mM EDTA, pH 7.4), followed by $10 \mu \mathrm{g}$ of cell lysate and incubated overnight at $4^{\circ} \mathrm{C}$. The wells were washed three times with $200 \mu \mathrm{l}$ washing buffer $(50 \mathrm{mM}$ Tris- $\mathrm{Cl}, 80 \mathrm{mM} \mathrm{NaCl}$, $0.5 \%$ BSA, $0.01 \%$ Tween-20, pH 7.4), followed by incubation with $100 \mu \mathrm{l}$ of $0.1 \mu \mathrm{g} / \mathrm{ml}$ streptavidin-conjugated Poly-HRP (Thermon Scientific, Rockford, Ill., USA) at room temperature for $1 \mathrm{~h}$. Subsequently, wells were washed three times with washing buffer and incubated in OPD solution $(0.6 \mathrm{mg} / \mathrm{ml}$ OPD, $35 \mathrm{~mm}$ citric acid, 50 $\mathrm{mM} \mathrm{Na}_{2} \mathrm{HPO}_{4}$, pH 5.0) containing $0.01 \%$ hydrogen peroxide for $30 \mathrm{~min}$ at room temperature. The reaction was stopped by adding $1 \mathrm{M} \mathrm{H}_{2} \mathrm{SO}_{4}$. Absorbance was measured on a microplate reader at a wavelength of $490 \mathrm{~nm}$ and was corrected for background, which was defined as the absorbance in cellular extracts obtained from experiments where BAP had been omitted from the culture medium.

\section{Calcium Deposition}

RVSMC were decalcified with $0.6 \mathrm{~N} \mathrm{HCl}$ for $24 \mathrm{~h}$. The calcium content of $\mathrm{HCl}$ supernatants was determined colorimetrically by the $o$-cresolphthalein complexone method (Calcium kit; Pointe Scientific) and normalized to protein content as previously described [9].

\section{Alkaline Phosphatase Activity}

ALP activity was measured using $p$-nitrophenyl substrate supplied in an alkaline phosphatase assay kit (Pointe Scientific) and normalized by protein content [9].

\section{Western Blotting}

The blots were incubated with antibody against TGM2 (1:1,000; Thermo-Fisher Scientific, Waltham, Mass., USA) overnight at $4^{\circ} \mathrm{C}$ followed by incubating with peroxidase-conjugated secondary antibody (1:5,000 dilution), and immunodetection with the Enhanced Chemiluminescence Kit (Amersham, Piscataway, N.J., USA). The band intensity was analyzed by ChemiDoc MP Imaging System (Imaging Lab 4.0, Bio-Rad, Richmond, Calif., USA) [9].

\section{Matrix Vesicle Isolation}

MVs were isolated by collagenase digestion [3]. Cells were incubated with crude collagenase $(500 \mathrm{U} / \mathrm{ml}$, type IA, Sigma) in a solution of $0.25 \mathrm{M}$ sucrose, $0.12 \mathrm{M} \mathrm{NaCl}, 0.01 \mathrm{M} \mathrm{KCl}$ and $0.02 \mathrm{M}$ Tris 
buffer, $\mathrm{pH} 7.45$, at $37^{\circ} \mathrm{C}$ for $3 \mathrm{~h}$. The digests were centrifuged at $800 \mathrm{~g}$ and 30,000 $\mathrm{g}$ to remove cell debris and microsomes, respectively. The supernatant was centrifuged at $250,000 \mathrm{~g}$ to pellet the MV followed by resuspension in TBS ( $\mathrm{pH} 7.6$ ) with $0.25 \mathrm{M}$ sucrose. The MV amount was determined by protein concentration (BioRad).

\section{MV-Collagen Calcification Assay}

Culture dishes were coated with type I collagen (Sigma) in a $0.01 \%$ solution in $0.1 \mathrm{M}$ acetic acid at room temperature for $4 \mathrm{~h}$, which yields approximately $8-10 \mu \mathrm{g} / \mathrm{cm}^{2}$ coating. MVs isolated as above were added in equal concentrations ( $10 \mu \mathrm{g} / \mathrm{dish})$ to type I collagen-coated culture dishes in the presence of calcification media (DMEM with $15 \%$ FBS, $5 \mathrm{mM} \beta$-glycerophosphate and $1 \mathrm{U} / \mathrm{ml}$ fetal ALP) to yield an acellular MV-collagen culture and incubated at $37^{\circ} \mathrm{C}$ for 6 days. To determine the magnitude of calcification of this MV-collagen culture, the media was removed and the MVECM complex on the culture dishes incubated in $0.6 \mathrm{~N} \mathrm{HCl}$ for $24 \mathrm{~h} \mathrm{[3].} \mathrm{The} \mathrm{calcium} \mathrm{content} \mathrm{of} \mathrm{HCl}$ supernatants was determined colorimetrically by the $o$-cresolphthalein complexone method (Calcium kit; Pointe Scientific).

\section{Cross-Linking of Fibronectin and Type I Collagen with TGM2} for $M V$-ECM Calcification

Cross-linked fibronectin or type I collagen were prepared by incubating $2 \mu \mathrm{g}$ of fibronectin (from bovine plasma; Sigma) or type I collagen (from rat tail; Sigma) with 2 mU of TGM2 (from guinea pig liver; Sigma) in $10 \mathrm{mM}$ Tris- $\mathrm{HCl}, \mathrm{pH} 8.3,3 \mathrm{mM} \mathrm{CaCl}_{2}$ and $1 \mathrm{mM}$ dithiothreitol (DTT) for $2 \mathrm{~h}$ at $37^{\circ} \mathrm{C}$ [12]. The same reaction was conducted without TGM2 as a control. The mixtures of proteins were then coated on culture dishes and dried overnight. MV were added to TGM2 cross-linked-coated or control-coated dishes and incubated with calcification media for 6 days and MV-ECM calcification determined as described above.

\section{Organ Culture}

Segments of rat thoracic aorta measuring $3 \mathrm{~cm}$ were harvested from 35-week-old CKD rats and gently cleared of surrounding tissues. Aortic segments were incubated in serum-free DMEM (Gibco, Long Island, N.Y., USA) containing penicillin/streptomycin at $37^{\circ} \mathrm{C}$ in a humidified $5 \% \mathrm{CO}_{2} / 95 \%$ incubator. Calcification was induced with the addition of $7.5 \mathrm{U} / \mathrm{ml}$ of calf intestinal ALP and $3.8 \mathrm{~mm}$ sodium phosphate (= calcification medium) according to the methods of Lomashvili et al. [13]. To determine the role of TGM2 in aorta calcification ex vivo, the TGM2 inhibitor cystamine (Sigma, St. Louis, Mo., USA) was added to the culture medium. After 7 days, aortic specimens were rinsed in normal saline, minced and decalcified in $300 \mu \mathrm{l}$ of $0.6 \mathrm{~N} \mathrm{HCl}$ for $72 \mathrm{~h}$ with gentle agitation. The calcium content of $\mathrm{HCl}$ supernatants was determined colorimetrically and normalized by tissue weight as previously described [5].

All procedures were reviewed and approved by the Indiana University School of Medicine Institutional Animal Care and Use Committee.

\section{Statistical Analysis}

Statistical analysis was conducted using ANOVA with Fisher's post hoc analysis. The results are expressed as means $\pm \mathrm{SD}$, with $\mathrm{p}<0.05$ considered significant (StatView, SAS Institute, Cary, N.C., USA).

Transglutaminase 2 and Vascular Calcification

\section{Results}

The Expression and Activity of TGM2 and RUNX2 Expression Is Increased in VSMC from CKD Rats Compared to That from Normal Rats

RVSMC isolated from CKD or normal rats were grown for 24 or $96 \mathrm{~h}$ and total RNA isolated. The results demonstrate that TGM2 expression by real-time PCR was increased at both 24 and $96 \mathrm{~h}$ in VSMC from CKD rats compared to cells isolated from normal rats (fig. 1a). To determine if TGM2 activity is altered in VSMC, VSMC from normal or CKD rats were grown for various times and cellular TGM2 activity measured. As shown in figure 1b, TGM2 activity is significantly increased at 48 and $96 \mathrm{~h}$ in VSMC from CKD rats compared to that from normal rats. Furthermore, the osteoblast transcription factor RUNX2 is also increased at $96 \mathrm{~h}$ in VSMC from $\mathrm{CKD}$ rats compared to normal rats but there is no difference at $24 \mathrm{~h}$ (fig. 1c). Thus, there is increased expression, followed by activity of TGM2, and then upregulation of the transcription factor RUNX2 indicative of a phenotypic shift of VSMC to osteoblast-like cells in VSMC from CKD rats.

\section{Inhibition of TGM2 Activity Decreased Calcification and Alkaline Phosphatase Activity in RVSMC from CKD Rats}

To determine the role of TGM2 in VSMC calcification, VSMC from CKD or normal rats were treated with calcification media in the presence or absence of various concentrations of cystamine for 10 days and calcification and ALP activity determined. The results demonstrated that calcification was significantly increased in VSMC from CKD rats compared to cells from normal rats (fig. 2a). Inhibition of TGM2 activity by cystamine dose-dependently reduced calcification in VSMC from CKD rats but had no effect on VSMC from normal rats (fig. 2a). Similarly, inhibition of TGM2 activity also decreased ALP activity in VSMC from CKD rats but had no effect on that from normal rats (fig. 2b). To further confirm the specificity of TGM2 inhibition, we also treated VSMC from $\mathrm{CKD}$ rats with calcification media in the presence or absence of Z006, a specific TGM2 inhibitor (Zedira GmbH, Darmstadt, Germany) [10]. The results demonstrated that inhibition of TGM2 activity with Z006 also significantly decreased the calcification of VSMC from CKD rats at 10 days (control: $1.24 \pm 0.10 \mu \mathrm{mol} / \mathrm{mg}$; Z006: 0.77 $\pm 0.19 \mu \mathrm{mol} / \mathrm{mg} ; \mathrm{p}<0.02$ ). These results suggest that increased TGM2 activity in CKD is involved in enhanced VSMC calcification and ALP activity. 


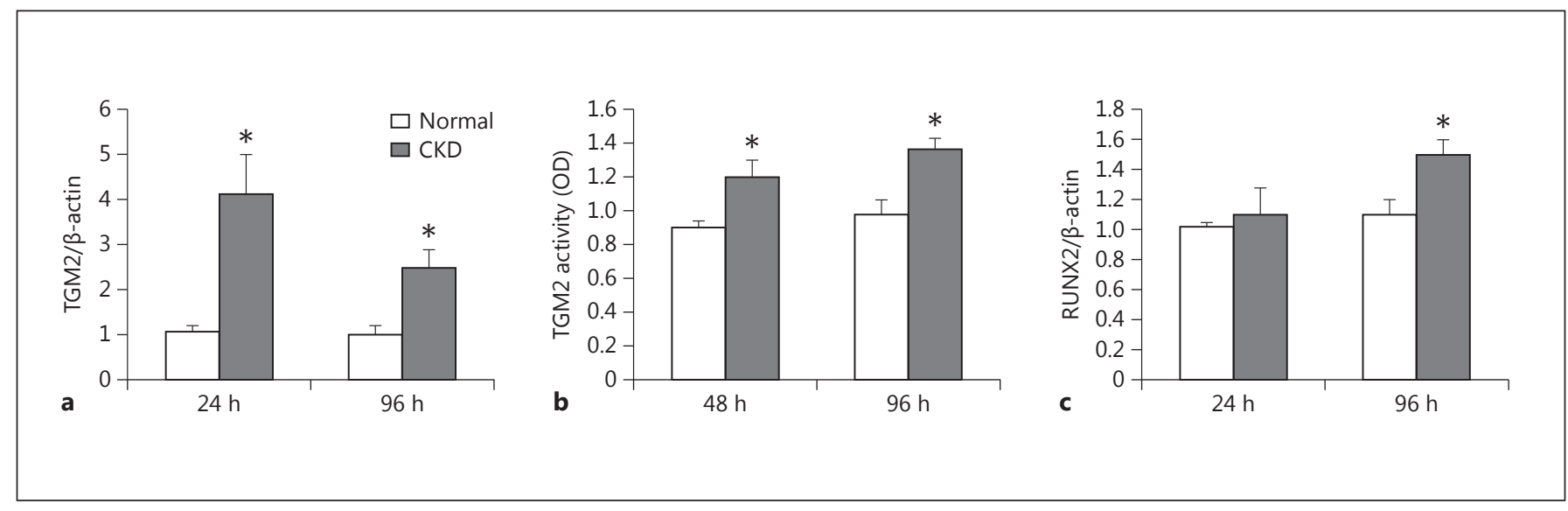

Fig. 1. The expression and activity of TGM2 and RUNX2 expression in VSMC from normal and CKD rats. VSMC isolated from normal or $\mathrm{Cy} /+(\mathrm{CKD})$ rats were incubated with growth media for 24 or $96 \mathrm{~h}$ and total RNA isolated for real time PCR for TGM2 and RUNX2 expression. The results demonstrate that TGM2 expression is increased at both 24 and $96 \mathrm{~h}$ in VSMC from CKD rats compared to that from normal rats (a). VSMC from normal or CKD rats were also grown for 48 or $96 \mathrm{~h}$ and cellular TGM2 activ- ity was determined. The results demonstrated that TGM2 activity is also increased in VSMC from CKD rats at both time points compared to that from normal rats (b). We also demonstrated that RUNX2 expression is increased at $96 \mathrm{~h}$ in VSMC from CKD rats compared to normal rats but there is no difference at $24 \mathrm{~h}$ (c). All experiments were run in triplicate. Data are shown as mean $\pm \mathrm{SD}$ (sample $\mathrm{n}=9$ ). ${ }^{*} \mathrm{p}<0.05, \mathrm{CKD}$ compared to normal.

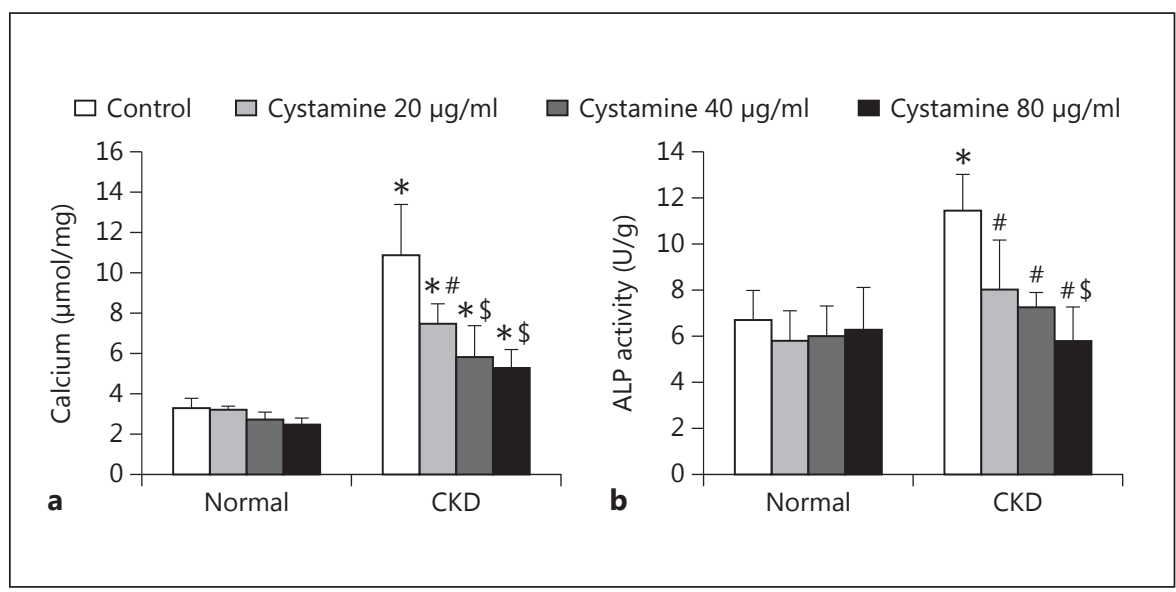

Fig. 2. The effect of the TGM2 inhibitor cystamine on calcification and ALP activity in VSMC from normal and CKD rats. VSMC from normal or CKD rats were treated with calcification media in the presence or absence of various concentrations of cystamine for 10 days and calcification and ALP activity determined. The results show calcification (a) and ALP activity (b) were significantly increased in VSMC from CKD rats compared to that from normal rats. Inhibition of TGM2 activity by cystamine dose-dependently reduced calcification (a) and ALP activity (b) in VSMC from CKD rats but had no effect on VSMC from normal rats. All experiments were run in triplicate. Data are shown as mean \pm SD (samples $n=$ 9). ${ }^{*} \mathrm{p}<0.05, \mathrm{CKD}$ vs. normal, no cystamine or with cystamine; ${ }^{\#} \mathrm{p}<0.05$, cystamine vs. control, CKD or normal; ${ }^{\$} \mathrm{p}<0.05$, highdose vs. low-dose cystamine, CKD or normal.
The Role of TGM2 in Matrix Vesicle Collagen Calcification in $C K D$

To determine the role of TGM2 in MV collagen calcification, we first isolated MV from CKD or normal RVSMC incubated in calcification media for 14 days and deter- mined the TGM2 content by Western blot. The results demonstrated that TGM2 is increased in MV from CKD RVSMC compared to that from normal RVSMC (fig. 3a). These MV were then added to type I collagen-coated dishes in our acellular calcification assay that assesses mineral- 


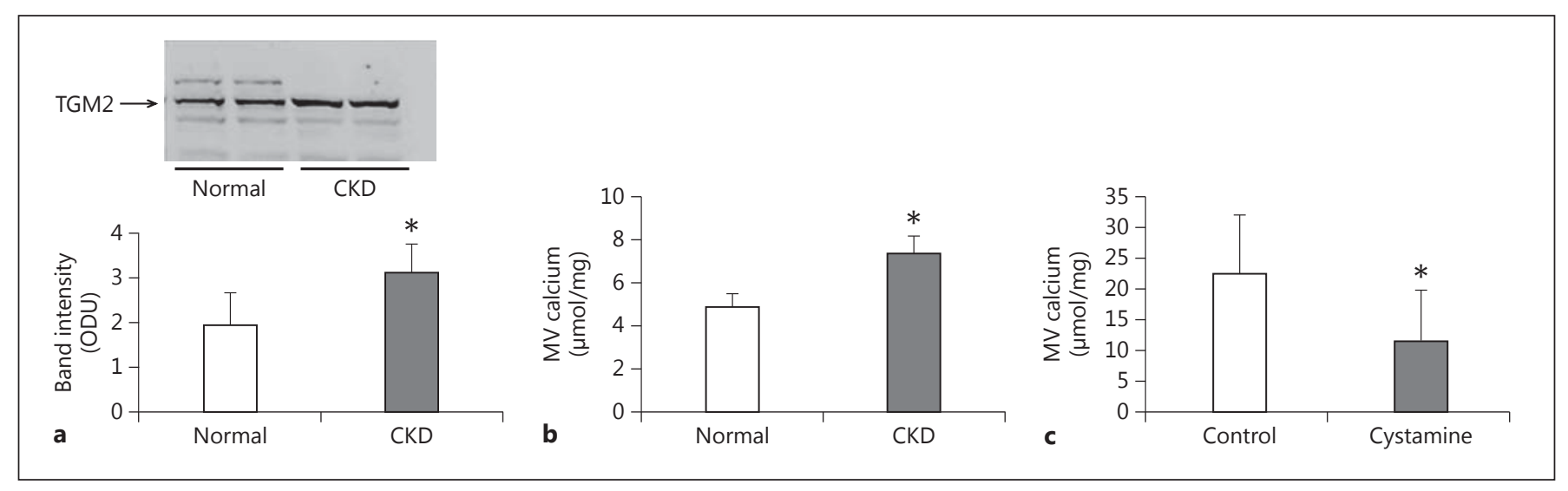

Fig. 3. The role of TGM2 in MV collagen calcification. VSMC from normal or CKD rats were incubated in calcification media $(5 \mathrm{mM}$ $\beta$-glycerophosphate, $1 \mathrm{U} / \mathrm{ml}$ fetal ALP) for 14 days and MV isolated and TGM 2 content determined by Western blot and band intensity was quantified. The results demonstrated that MV from VSMC derived from CKD rats contains high levels of TGM2 compared to that from normal rats (a). Ten micrograms of MV were then added to type I collagen-coated dishes in the presence of calcification media and incubated for 6 days and MV collagen calcification determined. The results demonstrated that MV from CKD RVSMC had increased calcification compared to that from normal

ization potential of the MV [3]. The results demonstrated that MV from CKD RVSMC had increased MV collagen calcification compared to that from normal RVSMC (fig. 3b). To further determine if TGM2 affects the ability of MV to calcify, MV were isolated from CKD RVSMC that had been incubated with calcification media in the absence (control) or presence of the TGM2 inhibitor cystamine. These MV were then added to type I collagen-coated dishes and calcification determined. The results demonstrate that inhibition of TGM2 activity during MV synthesis inhibited the ability of MV to subsequently mineralize on type I collagen (fig. 3c). This indicated that increased TGM2 activity in CKD is involved in MV-ECM mineralization.

\section{Cross-Linking of Fibronectin or Type I Collagen with}

TGM2 Enhanced the MV-ECM Calcification

To determine if cross-linking of ECM proteins by TGM2 may accelerate calcification, fibronectin or type I collagen was exogenously cross-linked by the addition of TGM2 and then coated on culture dishes. MV isolated from normal or CKD RVSMC were added and incubated with calcification media for 6 days and MV-ECM calcification determined. The calcification of MV on non-crosslinked fibronectin or type I collagen was greater in MV isolated from CKD RVSMC compared to normal cell-de-
RVSMC (b). To further determine the role of TGM2 in MV collagen calcification, MV were isolated from CKD RVSMC incubated with calcification media in the absence (control) or presence of cystamine $(40 \mu \mathrm{g} / \mathrm{ml})$ for 14 days. Ten micrograms of MV were added to type I collagen-coated dishes in the presence of calcification media and incubated for 6 days and MV collagen calcification determined. The results demonstrated that inhibition of TGM2 activity with cystamine during MV synthesis inhibited the ability of MV to mineralize on type I collagen (c). All experiments were run in triplicate. Data are shown as mean \pm SD (samples $n=6$ ). $* \mathrm{p}<0.05$, normal vs. CKD or control vs. cystamine.

rived MV (fig. 4). The exogenous cross-linking of fibronectin with TGM2 accelerated calcification in the CKD-, but not normal cell-derived MV. The exogenous crosslinking of type I collagen accelerated calcification in MV derived from both CKD and normal RVSMC (fig. 4). These results demonstrated that TGM2 cross-linking of ECM accelerates calcification.

\section{Inhibition of TGM2 Activity Decreased Aorta \\ Calcification ex vivo}

To confirm the importance of TGM2 in the pathogenesis of vascular calcification in CKD, we used the aorta ring culture model [13]. The aorta rings were isolated from CKD rats and incubated with calcification media in the presence or absence of theTGM2 inhibitor cystamine and aorta ring calcification determined. The results demonstrated that inhibition of TGM2 activity significantly decreased CKD rat aorta calcification (fig. 5).

\section{Discussion}

In the current study we have demonstrated a role of TGM2 in vascular calcification in CKD. Using the Cy/+ rat, a spontaneous and slowly progressive model of CKD- 


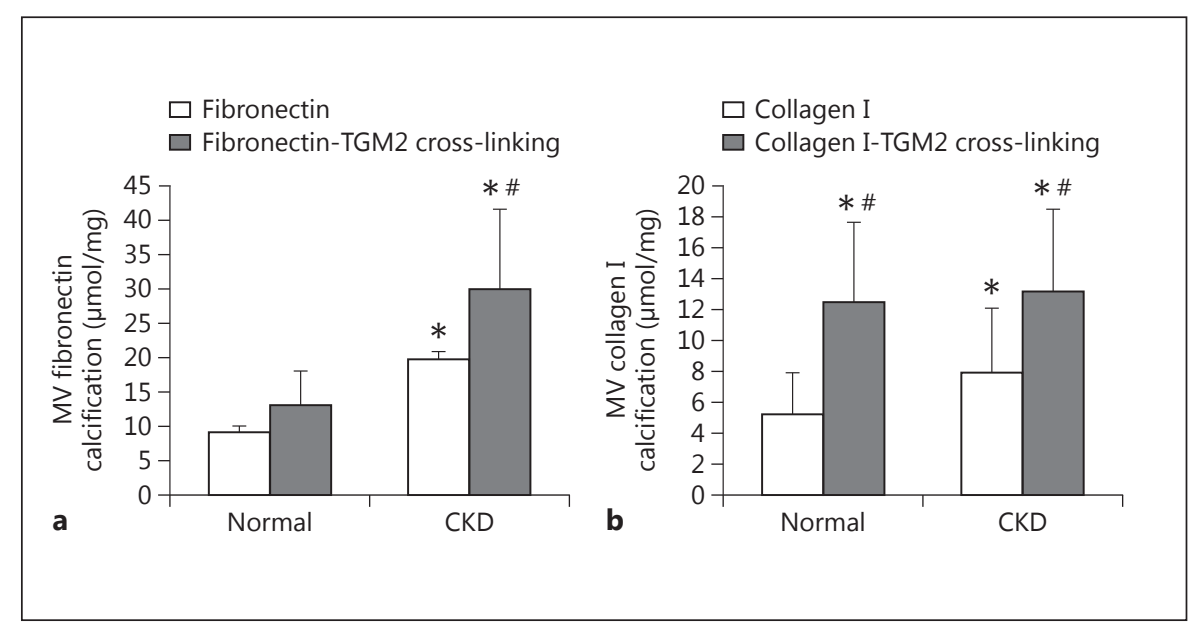

Fig. 4. Cross-linking of fibronectin or type I collagen with TGM2 enhanced the MV-ECM calcification. Fibronectin or type I collagen were cross-linked with TGM2 or without (control) and then used to coat 6-well plates culture plate. MV $(10 \mu \mathrm{g})$ isolated from normal or CKD RVSMC were added to these plates and incubated with calcification media for 6 days and MV-ECM calcification determined. The results demonstrated that MV fibronectin calcification is significantly increased in CKD rat-derived MV compared to that from normal rat MV (a). Furthermore, culture plates coated with fibronectin exogenously cross-linked by TGM2 increased

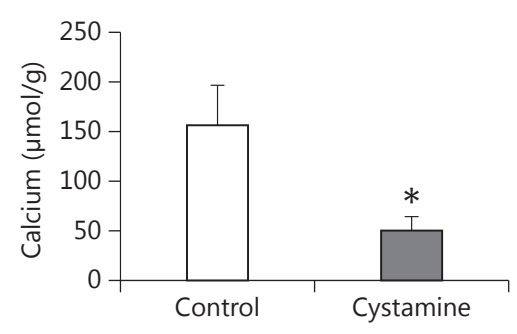

Fig. 5. Inhibition of TGM2 activity reduced ex vivo calcification of CKD rat aorta ring. Thoracic aorta rings isolated from CKD rats were incubated with calcification media in the presence of TGM2 inhibitors cystamine for 7 days and aorta calcification determined biochemically. The results demonstrated that inhibition of TGM2 activity significantly reduced aorta ring calcification. Data are shown as mean $\pm \mathrm{SD}$ ( $\mathrm{n}=3$ CKD rats). ${ }^{*} \mathrm{p}<0.05$ vs. controls.

mineral and bone disorder, we demonstrated that inhibition of TGM2 activity decreased arterial calcification in $\mathrm{CKD}$ rat-derived aortas in an ex vivo aorta ring culture model. We further demonstrated increased expression
CKD rat-derived MV calcification but had little effect on that from normal rat-derived MV (a). Similarly, type I collagen cross-linked by TGM2 also increased MV collagen calcification but had a similar effect in both CKD- and normal RVSMC-derived MV (b). These results confirm that one mechanism by which TGM2 increases calcification is by cross-linking the ECM to accelerate MV calcification. All experiments were run in triplicate. Data are shown as mean $\pm S D$ (samples $n=6){ }^{*} p<0.05$, CKD MV vs. normal MV, with or without TGM2 cross-linking; ${ }^{*} \mathrm{p}<0.05$, TGM2 cross-linking vs. no TGM2 cross-linking, normal MV or CKD MV.

and activity of TGM2 in VSMC from CKD rats compared to normal rats. The increased expression preceded an increased TGM2 enzyme activity and upregulation of the VSMC trans-differentiation factor RUNX2. Inhibition of TGM2 activity with cystamine decreased the calcification and ALP activity in cultured VSMC from CKD rats. Furthermore, MV derived from CKD RVSMC had increased TGM2 expression, and inhibition of TGM2 activity during MV formation impaired the ability of MV to calcify on type I collagen. We also demonstrated that TGM2 cross-linking of fibronectin or type I collagen promoted MV-ECM calcification. Thus, these data demonstrate that increased TGM2 activity in CKD may accelerate VSMC calcification by enhancing cross-linking of the ECM facilitating MV calcification. Based on the time course of observed changes in RUNX2, this occurs prior to the trans-differentiation of VSMC to osteoblast like cells and thus may be an initial step in the pathogenesis.

Transglutaminases are intracellular and extracellular enzymes that catalyze the calcium-dependent posttranslational modification of proteins. The enzymes catalyze modification of protein-bound glutamine side changes through transamidation or deamidation [14]. The reaction requires the presence of micromolar concentrations 
of calcium and thus can occur on the cell membrane at normal extracellular calcium concentrations, and achieved intracellular with calcium ionophores or calcium channel activation. TGM2 expression and enzyme activity is upregulated by retinoic acid, interleukin-6, tumor necrosis factor- $\alpha$ and is inhibited by bone morphogenic protein-2 and -4 [6]. TGM2 activity is also regulated by nitric oxide in the presence of calcium [15], and nitric oxide synthase inhibition leads to increased ECM TGM2. In the current study, we have shown that inhibition of TGM2 activity by cystamine decreased the calcification and ALP activity in VSMC from CKD rats. It should be pointed out that in addition to inhibition of TGM2 activity, cystamine is also potent inducer of antioxidants such as glutathione and L-cysteine in vivo and in vitro $[16,17]$. Furthermore, oxidative stress is known to be a risk factor for vascular calcification in CKD [18]. Our data support the primary role of cystamine in vascular calcification is through TGM2 as we also observed inhibition with a specific inhibitor of TGM2, Z006. TGM2 proteolysis is increased by matrix metalloproteinase (MMP)-2 and TGM2 upregulates MMP-2 [19] providing a regulatory feedback loop. Several studies have demonstrated the expression of TGM2 in bone, in areas of mineralized matrix near osteoblasts and osteocytes and in hypertrophic chondrocytes [20]. TGM2 substrates in bone include multiple collagen or non-collagenous proteins, including type I collagen, fibronectin, osteopontin, bone sialoprotein, and fetuin-A [20], with cross-linking promoting cell adhesion and migration $[12,21]$. TGM2 cross-linking of these proteins can stabilize the extracellular matrices rendering them resistant against proteolytic degradation [14].

Remodeling of arteries shares a common pathway to that in bone, where changes in ECM precede mineralization/calcification. Furthermore, inhibition of TGM2 activity reduced CKD aorta ring calcification. Our findings parallel studies in humans. In non-CKD human coronary arteries, TGM2 activity has been found in both the endothelium of the intima and medial VSMCs [6]. In atherosclerotic plaques, TGM2 is expressed predominately in areas of calcification [22]. TGM2 activity increases in aging rats, and inhibition of TGM2 activity removes the compensatory remodeling response to chronic vasoconstriction, decreased flow, and vascular stiffening [6]. Thus, TGM2 induces cross-linking of the ECM in the medial layer of arteries. It is likely that this cross-linking is initially stabilizing, but if it continues it may become pathologic and induce arterial stiffening [23]. The latter is associated with cardiovascular disease and arterial calcification in CKD patients [24]. We have previously also identified altered regulation of MMPs as another factor that predisposes to ECM remodeling, facilitating arterial calcification in CKD [25]. Thus, as an initiating step in arterial calcification, the increase in TGM2 and MMPs may remodel the medial layer ECM by degradation through metalloproteinase and cross-linking by TGM2, thus 'priming' the ECM for subsequent mineralization.

Our earlier studies demonstrated that VSMC-derived MVs serve as initiators of mineralization [3]. The interaction between MV and the ECM is modifiable as regulation can occur prior to MV formation by altering the cell culture conditions, or after MV formation by altering the interaction of the MV with the ECM. We and other groups also demonstrated that in the presence of phosphorus, MV can calcify ECM, and other factors in MV such as calcium, annexins and fetuin-A can affect this process $[3,26]$. In the current study, we also demonstrated there is an increased TGM2 content in MV derived from CKD RVSMC, and inhibition of TGM2 activity during MV synthesis impaired the ability of MV to calcify on type I collagen. Furthermore, TGM2 cross-linking of fibronectin or type I collagen promote MV-ECM calcification. TGM2 has been reported to act as a $\beta_{1^{-}}$and $\beta_{3^{-}}$ integrin-binding co-receptor for fibronectin [27]. Thus, blocking the MV TGM2 activity may affect integrin-mediated binding to the ECM. Whether this is due to intracellular TGM2, and/or membrane TGM2 and if extracellular calcium plays a role is currently under investigation.

When non-CKD VSMC are cultured on type I collagen that is cross-linked with exogenous TGM2, the cells demonstrate increased spreading, proliferation and contractile response [23]. Cross-linking of fibronectin by TGM2 not only activates integrin-mediated ECM binding but also induces other signaling pathways such as RhoA [28]. We have previously demonstrated that RhoA activity can modulate fetuin-A uptake and VSMC and MV calcification [5]. Our data demonstrates that when fibronectin or type I collagen are exogenously crosslinked by TGM2, the enzymatic process accelerates MV calcification on the ECM. Thus, the cross-linking alters cellular phenotype and MV calcification. VSMC from TGM $2^{-/-}$mice also had increased expression of calcification inhibitors such as matrix gla protein and osteopontin, suggesting that the cross-linking of these proteins lessens their inhibitory effects [8]. Thus, the cross-linking of the ECM by TGM2 may be an important step in mineralization by inducing a phenotypic switch of VSMC to contractile 'osteoblast-like' cells. These cells then make MV, which calcify more on a TGM2 cross-linked ECM. Finally, the calcification is further propagated by cross- 
linking, and therefore reducing function of calcification inhibitors.

In summary, we have demonstrated a potential role for TGM2 in the pathogenesis of vascular calcification in CKD. The ability of TGM2 to cross-link the ECM leads to enhanced cell-ECM and MV-ECM attachment, increased cell proliferation and trans-differentiation, and cross-linking various inhibitors of calcification. Our re- sults suggest that blocking TGM2 activity may lead to reduced vascular calcification in CKD and therefore could offer therapeutic benefit.

\section{Acknowledgement}

This work was supported by a VA Merit Award and NIH R01 (S.M.M.).

\section{References}

$\checkmark 1$ Moe SM, Chen NX: Mechanisms of vascular calcification in chronic kidney disease. J Am Soc Nephrol 2008;19:213-216.

-2 Moe SM, Chen NX: Pathophysiology of vascular calcification in chronic kidney disease. Circ Res 2004;95:560-567.

$\checkmark 3$ Chen NX, O’Neill KD, Chen X, Moe SM: Annexin-mediated matrix vesicle calcification in vascular smooth muscle cells. J Bone Miner Res 2008;23:1798-1805.

$\checkmark 4$ Chen NX, Kircelli F, O’Neill KD, Chen X, Moe SM: Verapamil inhibits calcification and matrix vesicle activity of bovine vascular smooth muscle cells. Kidney Int 2010;77:436442 .

5 Chen NX, Chen X, O’Neill KD, Atkinson SJ, Moe SM: RhoA/Rho kinase (ROCK) alters fetuin-A uptake and regulates the calcification in bovine vascular smooth muscle cells (BVSMC). Am J Physiol Renal Physiol 2010; 299:F674-F680.

6 Bakker EN, Pistea A, VanBavel E: Transglutaminases in vascular biology: relevance for vascular remodeling and atherosclerosis. J Vasc Res 2008;45:271-278.

$>7$ Al-Jallad HF, Nakano Y, Chen JL, McMillan E, Lefebvre C, Kaartinen MT: Transglutaminase activity regulates osteoblast differentiation and matrix mineralization in MC3T3-E1 osteoblast cultures. Matrix Biol 2006;25:135148.

$>8$ Johnson KA, Polewski M, Terkeltaub RA: Transglutaminase 2 is central to induction of the arterial calcification program by smooth muscle cells. Circ Res 2008;102:529-537.

$\checkmark 9$ Chen NX, O’Neill KD, Duan D, Moe SM: Phosphorus and uremic serum up-regulate osteopontin expression in vascular smooth muscle cells. Kidney Int 2002;62:1724-1731.

10 Schaertl S, Prime M, Wityak J, Dominguez C, Munoz-Sanjuan I, Pacifici RE, Courtney S, Scheel A, Macdonald D: A profiling platform for the characterization of transglutaminase 2 (TG2) inhibitors. J Biomol Screen 2010;15: 478-487.
11 Verhaar R, Jongenelen CA, Gerard M, Baekelandt V, Van Dam AM, Wilhelmus MM, Drukarch B: Blockade of enzyme activity inhibits tissue transglutaminase-mediated transamidation of alpha-synuclein in a cellular model of Parkinson's disease. Neurochem Int 2011; 58:785-793.

12 Forsprecher J, Wang Z, Nelea V, Kaartinen MT: Enhanced osteoblast adhesion on transglutaminase 2-crosslinked fibronectin. Amino Acids 2009;36:747-753.

13 Lomashvili KA, Cobbs S, Hennigar RA, Hardcastle KI, O’Neill WC: Phosphate-induced vascular calcification: role of pyrophosphate and osteopontin. J Am Soc Nephrol 2004;15: 1392-1401.

14 Lorand L, Graham RM: Transglutaminases: crosslinking enzymes with pleiotropic functions. Nat Rev Mol Cell Biol 2003;4:140-156.

15 Lai TS, Hausladen A, Slaughter TF, Eu JP, Stamler JS, Greenberg CS: Calcium regulates S-nitrosylation, denitrosylation, and activity of tissue transglutaminase. Biochemistry 2001;40:4904-4910.

- 16 Fox JH, Barber DS, Singh B, Zucker B, Swindell MK, Norflus F, Buzescu R, Chopra R, Ferrante RJ, Kazantsev A, Hersch SM: Cystamine increases L-cysteine levels in Huntington's disease transgenic mouse brain and in a PC12 model of polyglutamine aggregation. J Neurochem 2004;91:413-422.

17 Takano K, Shiraiwa K, Moriyama M, Nakamura Y: Transglutaminase 2 expression induced by lipopolysaccharide stimulation together with NO synthase induction in cultured astrocytes. Neurochem Int 2010;57: 812-818.

18 Chen NX, Moe SM: Vascular calcification: pathophysiology and risk factors. Curr Hypertens Rep 2012;14:228-237.

19 Munezane T, Hasegawa T, Suritala, Tanaka A, Okada K, Okita Y: Activation of transglutaminase type 2 for aortic wall protection in a rat abdominal aortic aneurysm formation. J Vasc Surg 2010;52:967-974.
20 Kaartinen MT, El-Maadawy S, Rasanen NH, McKee MD: Tissue transglutaminase and its substrates in bone. J Bone Miner Res 2002;17: 2161-2173.

21 Forsprecher J, Wang Z, Goldberg HA, Kaartinen MT: Transglutaminase-mediated oligomerization promotes osteoblast adhesive properties of osteopontin and bone sialoprotein. Cell Adh Migr 2011;5:65-72.

22 Matlung HL, Groen HC, de Vos J, van Walsum T, van der Lugt A, Niessen WJ, Wentzel JJ, Vanbavel E, Bakker EN: Calcification locates to transglutaminases in advanced human atherosclerotic lesions. Am J Pathol 2009; 175:1374-1379.

23 Spurlin TA, Bhadriraju K, Chung KH, Tona A, Plant AL: The treatment of collagen fibrils by tissue transglutaminase to promote vascular smooth muscle cell contractile signaling. Biomaterials 2009;30:5486-5496.

24 Guerin AP, London GM, Marchais SJ, Metivier F: Arterial stiffening and vascular calcifications in end-stage renal disease. Nephrology, Dialysis, Transplantation 2000;15:10141021.

25 Chen NX, O’Neill KD, Chen X, Kiattisunthorn K, Gattone VH, Moe SM: Activation of arterial matrix metalloproteinases leads to vascular calcification in chronic kidney disease. Am J Nephrol 2011;34:211-219.

26 Kapustin AN, Davies JD, Reynolds JL, McNair R, Jones GT, Sidibe A, Schurgers LJ, Skepper JN, Proudfoot D, Mayr M, Shanahan CM: Calcium regulates key components of vascular smooth muscle cell-derived matrix vesicles to enhance mineralization. Circ Res 2011;109:e1-e12.

27 Akimov SS, Krylov D, Fleischman LF, Belkin AM: Tissue transglutaminase is an integrinbinding adhesion coreceptor for fibronectin. J Cell Biol 2000;148:825-838.

28 Janiak A, Zemskov EA, Belkin AM: Cell surface transglutaminase promotes RhoA activation via integrin clustering and suppression of the Src-p190RhoGAP signaling pathway. Mol Biol Cell 2006;17:1606-1619. 